\title{
Musical Auditory Alpha Wave Neurofeedback: Validation and Cognitive Perspectives
}

\author{
Kazuhiko Takabatake ${ }^{1} \cdot$ Naoto Kunii $^{1} \cdot$ Hirofumi Nakatomi $^{1}$ (D ) Seijiro Shimada ${ }^{1} \cdot$ Kei Yanai $^{1} \cdot$ Megumi Takasago $^{1}$. \\ Nobuhito Saito ${ }^{1}$
}

Accepted: 2 April 2021 / Published online: 30 April 2021

(c) The Author(s) 2021

\begin{abstract}
Neurofeedback through visual, auditory, or tactile sensations improves cognitive functions and alters the activities of daily living. However, some people, such as children and the elderly, have difficulty concentrating on neurofeedback for a long time. Constant stressless neurofeedback for a long time may be achieved with auditory neurofeedback using music. The primary purpose of this study was to clarify whether music-based auditory neurofeedback increases the power of the alpha wave in healthy subjects. During neurofeedback, white noise was superimposed on classical music, with the noise level inversely correlating with normalized alpha wave power. This was a single-blind, randomized control crossover trial in which 10 healthy subjects underwent, in an assigned order, normal and random feedback (NF and RF), either of which was at least 4 weeks long. Cognitive functions were evaluated before, between, and after each neurofeedback period. The secondary purpose was to assess neurofeedback-induced changes in cognitive functions. A crossover analysis showed that normalized alpha-power was significantly higher in NF than in RF; therefore, music-based auditory neurofeedback facilitated alpha wave induction. A composite category-based analysis of cognitive functions revealed greater improvements in short-term memory in subjects whose alpha-power increased in response to NF. The present study employed a long period of auditory alpha neurofeedback and achieved successful alpha wave induction and subsequent improvements in cognitive functions. Although this was a pilot study that validated a music-based alpha neurofeedback system for healthy subjects, the results obtained are encouraging for those with difficulty in concentrating on conventional alpha neurofeedback.
\end{abstract}

Trial registration: 2018077NI, date of registration: 2018/11/27

Keywords Neurofeedback $\cdot$ Alpha wave $\cdot$ Wearable EEG $\cdot$ Short-term memory $\cdot$ Working memory

\section{Introduction}

Neurofeedback is a method to self-regulate brain function by measuring brain activity and presenting it to subjects. Brain activity is assessed using various modalities, such as functional magnetic resonance imaging (fMRI) (Sulzer et al., 2013), near-infrared spectroscopy (NIRS) (Mihara et al., 2012), scalp electroencephalogram (EEG) (Zoefel et al., 2011), and intracranial EEG (Yamin et al., 2017). One of the most widely utilized methods of neurofeedback involves the alpha wave from EEG (Yeh et al., 2020).

Hirofumi Nakatomi

hnakatomi-tky@umin.ac.jp

1 Department of Neurosurgery, The University of Tokyo, 7-3-1 Hongo, Bunkyo-ku, Tokyo 113-8655, Japan
The alpha wave is an $8-13 \mathrm{~Hz}$ brainwave that is mainly observed in the occipital region of healthy individuals and is enhanced by closing the eyes. The augmentation of alpha waves by neurofeedback may mitigate the symptoms of psychiatric disorders, such as anxiety and depression (Markiewcz, 2017; Schoenberg \& David, 2014; Schönenberg et al., 2017). An increasing number of studies have reported the usefulness of alpha wave feedback in patients with attention-deficit and hyperactivity disorder (ADHD) and emphasized its importance in clinical settings (Lofthouse et al., 2012). However, some patients with ADHD cannot concentrate on alpha neurofeedback for an entire session, resulting in varying rates of drop-outs (Duric et al., 2012; Gevensleben et al., 2010). Previous studies mostly adopted a neurofeedback protocol in which the amplitude of alpha wave power was visually presented (Biswas \& Ray, 2019; Choi et al., 2011; Escolano et al., 
2014; Gruzelier, 2014; Hsueh et al., 2016; Lavy et al., 2019). Subjects need to spend the entire time concentrating on a monitor, which may be intolerable for some children and the elderly as well as patients with ADHD. Since the framework of alpha neurofeedback requires the continuous participation of subjects in repeated sessions, a novel protocol in which subjects may relax during the session needs to be developed.

Previous studies on auditory alpha neurofeedback reported the augmentation of the alpha wave as well as improvements in cognitive function (Bucho et al., 2019; Cho et al., 2008; Fernández et al., 2016; van Boxtel et al., 2012). However, these studies adopted monotonous auditory stimulations, which may not be relaxing subjects during neurofeedback sessions. The use of music may overcome this issue. Music has been used to improve various neurological conditions. Recently, biofeedback using sonification has been shown to be effective and is attracting a growing attention (Bergstrom et al., 2014; Brancatisano et al., 2020; Fedotchev et al., 2018; Maes et al., 2016). However, there are only a limited number of studies reporting that combining such music therapy and alpha neurofeedback has neuroprotective effect (Alexander, 2018; Nawaz et al., 2018; Ramirez et al., 2015). Prior to the introduction of music-based alpha neurofeedback to individuals with difficulty maintaining concentration, it is important to validate whether the protocol effectively induces the alpha wave and clarify its effects on cognitive functions in healthy subjects.

Therefore, in the present study, healthy subjects underwent more than two-month-long sessions of neurofeedback using ALPHA SWITCH ver.0.9.0 (Mediaseek Inc., Tokyo, Japan, https://www.mediaseek.co.jp/alpha-switch/, available on App Store), which is an application developed to provide an opportunity to experience auditory alpha-neurofeedback while listening to music. This system appears to free subjects from the stress of concentrating on a monitor with their eyes open, thereby reducing the burden on subjects to keep participating in long-term neurofeedback. This system is also almost free of EEG noises generated by eye movement and eye opening/closing, which is always a major issue in scalp EEG recordings because subjects are allowed to close their eyes during each session (Jebelli et al., 2018).

The primary purpose of the present study was to validate whether the novel auditory neurofeedback system augments the power of the alpha wave in healthy subjects. The secondary purpose was to compare changes in cognitive functions evaluated before, between, and after each feedback. The present study was a single-blind, randomized control crossover trial, which enables a more efficient comparison than a parallel design with fewer subjects because each subject serves as his/her own matched control.

\section{Methods}

\section{Subjects}

Subjects were required to be present for measurements in our facility for approximately $15 \mathrm{~min}$ per day for 3-4 days a week over two months. Ten doctors from the Department of Neurosurgery of the University of Tokyo participated in the present study. All subjects were male and the average age was 33.8 years (31-36 years).

No subjects had previously experienced neurofeedback, had taken any medications for the nervous system, or had a history of neurological or mental diseases. The present study was approved by the Research Ethics Committee, Graduate School of Medicine and Faculty of Medicine, the University of Tokyo. All subjects provided written informed consent.

\section{EEG Device}

Muse (InteraXon Inc., Toronto, Canada, https://choos emuse.com/muse-2/) is a headband-type wearable EEG device that is mounted on the forehead with the end of the band held on each ear. Although EEG may be easily measured without a special pretreatment on the scalp with this device, we applied EEG paste on the scalp for recording in the present study. Muse has 4 active electrodes and one reference electrode. Two active electrodes made of silver chloride are located on the bilateral forehead and two other electrodes made of conductive silicon rubber are on the dorsal side of the bilateral auricles. The reference electrode is located between the two active electrodes on the forehead. The sampling rate was fixed at $256 \mathrm{~Hz}$. Recorded data was transferred to the tablet device (iPad, Apple Inc., California, The United States of America) via Bluetooth without a delay. EEG data recorded by the four electrodes of Muse were saved in the respective four channels of the tablet device after being processed by a $50-\mathrm{Hz}$ notch filter.

\section{Schedule of the Feedback Study}

The present study was designed as a crossover study, in which each subject went into the first and second feedback periods using normal feedback (NF) or random feedback (RF) in an assigned order (Fig. 1). Each subject was assigned to group A or B, each of which had 5 subjects. Subjects in group A underwent NF in the first feedback period and RF in the second feedback period, while subjects in group B received each feedback in the reverse order. The first and second feedback periods were 


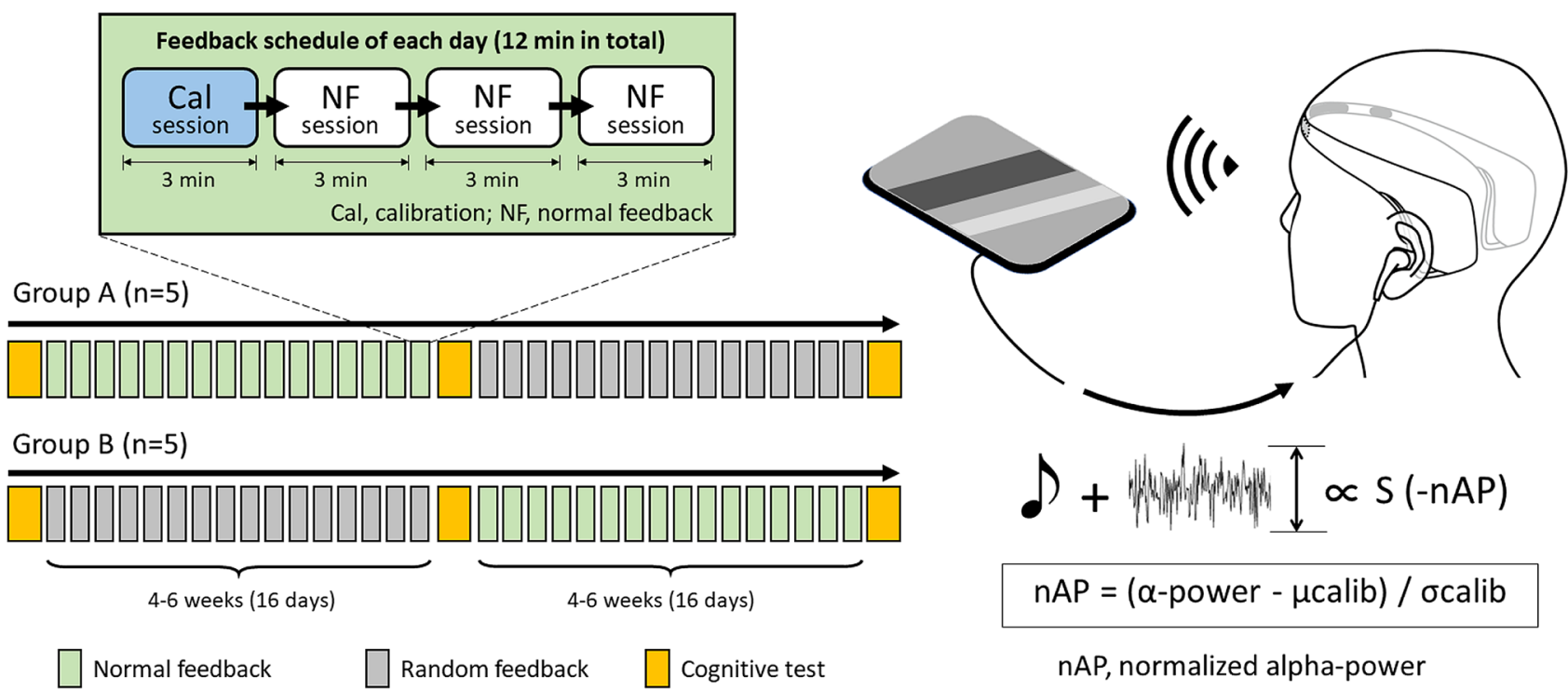

Fig. 1 A schema of the schedule of neurofeedback in this study (left) and the adopted neurofeedback system (right). The subjects of group A underwent $\mathrm{NF}$ in the first feedback period and RF in the second feedback period, while subjects of group B underwent each feedback in reverse order. In the normal neurofeedback, the subject listened to classical music with the eyes closed and Muse mounted on the head. The superimposed white noise was updated every three seconds

separated by an interval of at least two days. Subjects were blind to which group they were assigned to and which feedback they were receiving.

In every feedback period, each subject was engaged in feedback sessions for three to four days a week depending on subject availability, resulting in each feedback period of 16 days ranging between four and six weeks. The subject performed one calibration session and three feedback sessions consecutively per day in a feedback period. We postponed the measurement when subjects felt too tired or drowsy to maintain their concentration for $15 \mathrm{~min}$ of feedback. In groups A and B, the cognitive functions of all subjects were evaluated three times; before, between, and after each feedback period.

\section{Neurofeedback}

The subject was seated in a quiet room with their eyes closed and Muse mounted on the head. The recording and real time analysis of EEG and real time feedback of the analyzed result were performed using default function of ALPHA SWITCH as follows. Before the feedback session, EEG was recorded for $180 \mathrm{~s}$ for calibration, during which the reliability of the recording was checked by referring to the power law distribution of physiological EEG (Namazi \& Kulish, 2012; van Albada \& Robinson, 2013). Every three-second data set of each channel was processed by an $8-13-\mathrm{Hz}$ bandpass so that the noise level inversely correlated to the alpha-power, normalized to that of the calibration session. $N F$ normal feedback, $R F$ random feedback, Cal calibration, $\alpha$-power averaged alpha power in feedback session for every three second, $\mu c a l i b$ averaged alpha power in calibration session, ocalib standard deviation of alpha power in calibration session

filter and Hilbert transformation, yielding power in the alpha range (alpha-power). Averaged alpha-power was obtained every $3 \mathrm{~s}$. Data was analyzed and output in real time by the application installed in the tablet device. Fast Fourier transformation was applied for every three seconds of EEG data and calculated the power spectral density curve. Using the least-squares method, the slope of the curve was calculated between $1 \mathrm{~Hz}$ and the Nyquist frequency. Three-second data with a slope between -0.30 and -0.14 were omitted due to excessive noise, and recordings were performed again when the omitted data surpassed $5 \%$ of all calibration data. The Smirnov-Grubbs test was used to exclude outliers $(p<0.05)$ from calibration data. The time average ( $\mu$ calib) and standard deviation ( $\sigma$ calib) of alpha-power were calculated.

A feedback session for $180 \mathrm{~s}$ followed the calibration session. Durations of feedback sessions in the previous studies on alpha neurofeedback range between 3 and 30 min (Jirayucharoensak et al., 2019; Naas et al., 2019; Wei et al., 2017). We adopted this short duration in the present study to avoid sleeping during the feedback session with subjects' eyes closed. Each subject underwent NF or RF according to the assignment. In the same manner as the calibration session, the time average of alpha-power was calculated every three seconds, and was then converted to z-scores of alphapower using $\mu$ calib and $\sigma$ calib. The normalized alpha-power (nAP) of each three-second data was obtained by averaging the z-scores of four channels. 
Feedback was delivered to each subject auditorily via earphones while listening to classical music, "Air on the G String" (Fig. 1). In NF, white noise was superimposed such that the noise level inversely correlated with nAP. Along with the sigmoid function, the volume of white noise was set to zero and the maximum when nAP was 2 and -2 , respectively. The maximum volume levels of music and white noise were approximately $60-70$ and $60 \mathrm{~dB}$, respectively. The subject was instructed to minimize the noise level by increasing alpha-power as much as possible. In RF, the volume of white noise superimposed onto music varied in the same range as NF according to the randomly mixed EEG data of healthy volunteers. Therefore, subjects listened to a prerecorded sound during RF, while the recoding of EEG was performed using ALPHA SWITCH (Fig. 2).

\section{Data Analysis of Neurofeedback}

Data recorded during feedback sessions were extracted from the tablet and analyzed offline using custom-made Matlab (The Mathworks, Natick, USA) programs. Previous studies on alpha wave neurofeedback reported that it took two weeks or 8 days of feedback before a significant difference was noted between the feedback and control groups (Lau-Zhu et al., 2019). Based on these findings, the impact of neurofeedback in the present study was assumed to be detectable in the late half of each feedback period. Each feedback period was divided into an early section (1-8 days) and late section (9-16 days) and the following analysis was applied in the late section. Timeaveraged nAPs were averaged among three sessions in each day, yielding daily nAP. Since noisy nAPs were not omitted during feedback sessions, we excluded the maximum and minimum as outliers from the 8 daily nAPs of each section. Then, the average of 6 daily nAPs, which represented the section, was calculated. The daily nAPs of the late section of the first period in group A and the late section of the second period in group B were combined to represent the daily nAPs of NF across the groups. Similarly, the daily nAPs of the late section of the second period in group $\mathrm{A}$ and the late section of the first period in group B represented the daily nAPs of RF across the groups (Fig. 3a). The daily nAPs of NF and RF were statistically compared using a paired $t$-test $(\alpha=0.05)$. Before the comparison, the normality of the distribution was tested using the Shapiro-Wilk test.

In addition to the above analysis, a robust regression analysis of 16 daily nAPs in the NF period was performed for each subject. Subjects were classified into responders or non-responders depending on whether the regression coefficient was positive or not, respectively. We then examined the association between responsiveness of NF and cognitive test scores, as described below.

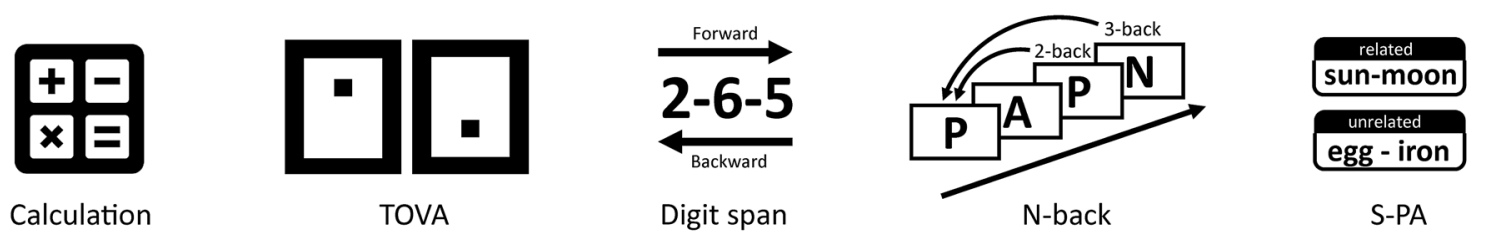

\begin{tabular}{lcccc}
\hline Composite category & Attention & $\begin{array}{c}\text { Short-term } \\
\text { memory }\end{array}$ & $\begin{array}{c}\text { Working } \\
\text { memory }\end{array}$ & $\begin{array}{c}\text { Verbal } \\
\text { memory }\end{array}$ \\
\hline Calculation & $\checkmark$ & & & \\
TOVA & $\checkmark$ & & & \\
Digit span (forward) & $\checkmark$ & $\checkmark$ & & \\
Digit span (backward) & $\checkmark$ & $\checkmark$ & $\checkmark$ & $\checkmark$ \\
2-back & $\checkmark$ & $\checkmark$ & $\checkmark$ & $\checkmark$ \\
3-back & $\checkmark$ & $\checkmark$ & $\checkmark$ & \\
S-PA (related) & & & & \\
\hline S-PA (unrelated) & & & & \\
\hline
\end{tabular}

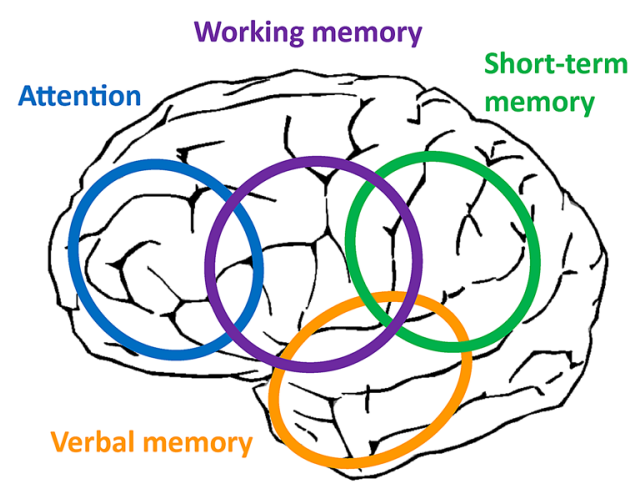

Fig. 2 Cognitive tests and the corresponding brain functions. Eight cognitive tests derived from 5 tasks were used in this study. Four composite categories were generated based on the function each cognitive test was designed to evaluate. The right lower figure illustrates the rough area of the epicenter of each function. TOVA The Test of Variables of Attention, S-PA Standard verbal Paired Associate Learning Test 
a

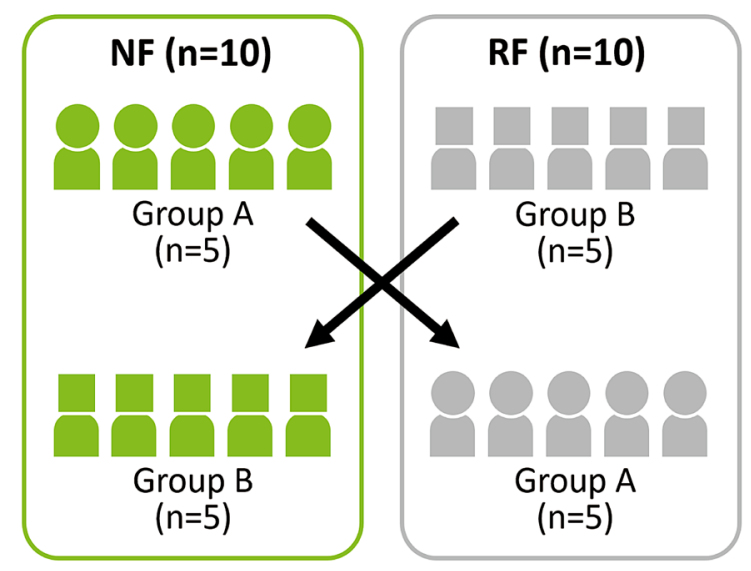

C

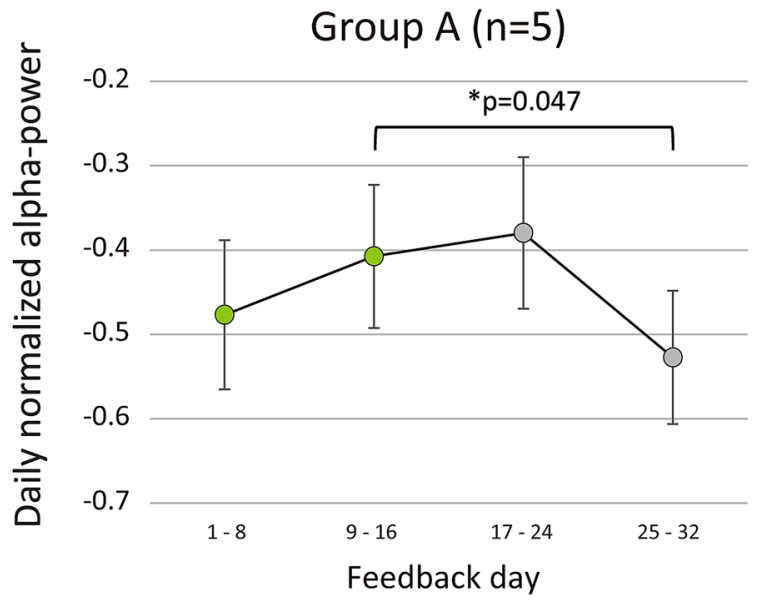

Fig. 3 Comparison of the normalized alpha-power between normal and random neurofeedback. A schema of comparison in a crossover design (a). Yellow-green and gray colors refer to each subject in normal and random neurofeedback (NF and RF), respectively. Comparison between NF and RF regardless of the groups (b). The daily normalized alpha-power in NF was significantly higher than that in RF

\section{Cognitive Tests}

Cognitive functions were evaluated three times; before, between, and after each feedback period. Each subject was assessed using the digit span test (DST), standard verbal paired-associate learning test (S-PA), simple calculation task (SCT), N-back test (NBT), and Test of Variables of Attention (TOVA) in this order. The total duration of all cognitive tasks was approximately $45 \mathrm{~min}$.

In DST, the subject listened to a series of digits that were read out by an examiner at a pace of one digit per second
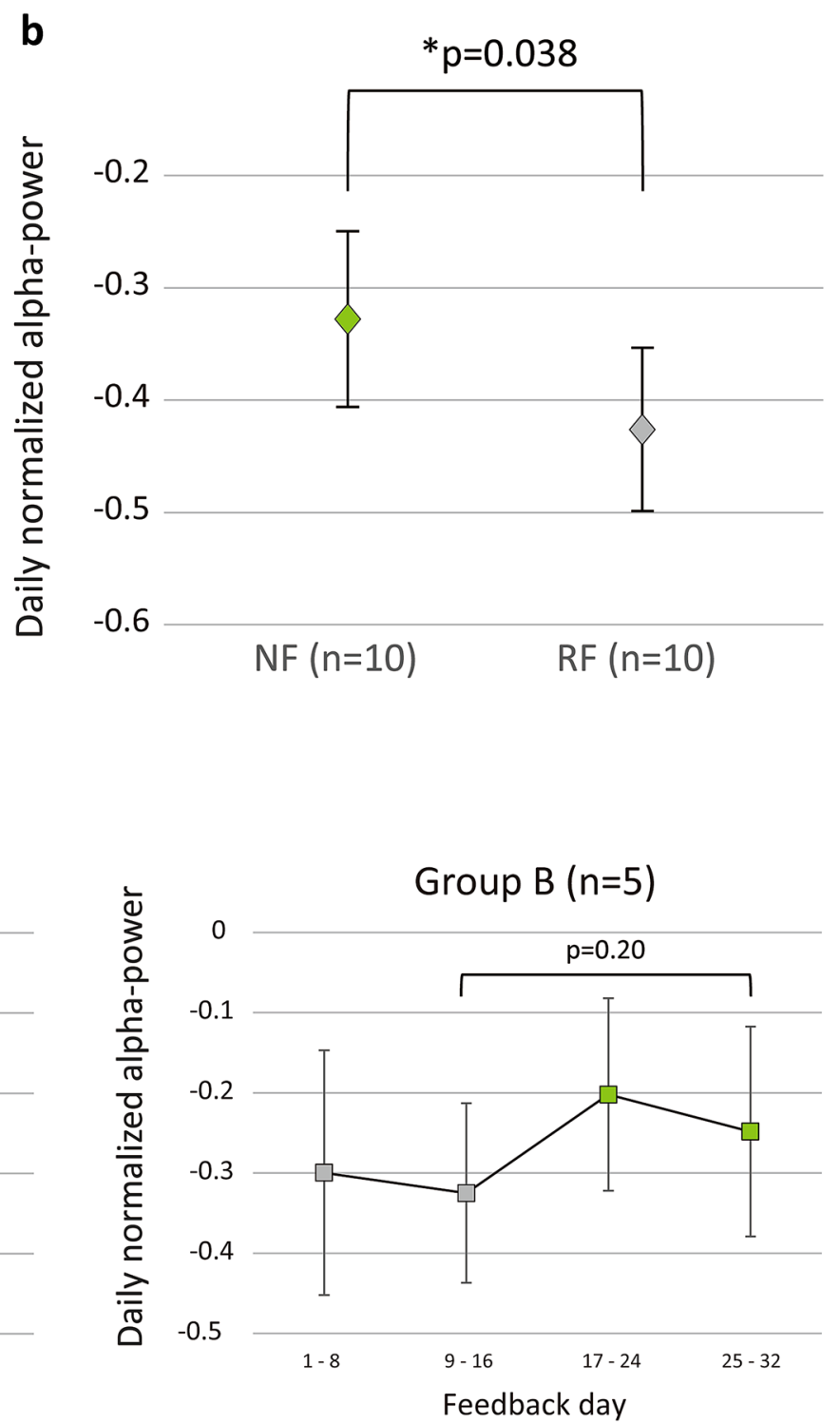

$(p=0.0383)$. The time courses of the daily normalized alpha-power are shown separately for group A and B (c). Group A showed a slight increase in NF (yellow-green) and a decrease in RF periods (gray). The daily normalized alpha-power of the late section was significantly higher in NF than RF period $(p=0.047)$

and was asked to repeat them in the same (forward DST) or reverse order (backward DST) as presented. The digit number was increased one by one before two consecutive errors were made. The maximum digit number was the score of each task (Giofre et al., 2016; Woods et al., 2011).

In S-PA, the subject listened to and remembered a series of word pairs that were read out by an examiner at a pace of one word per second. The examiner then read out one word of each pair and the subject was asked to respond with the other word of the pair. S-PA consists of three batteries, each of which has two sets of ten pairs of Japanese words 
a

90909

Group A

$(n=5)$

0000

Responder

$(n=6)$

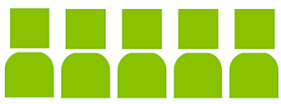

Group B

$(n=5)$

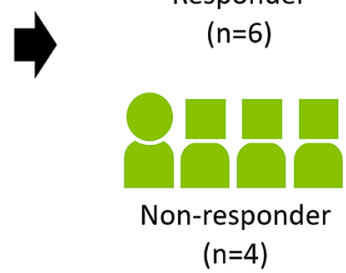

b

Responsder

Non-responder

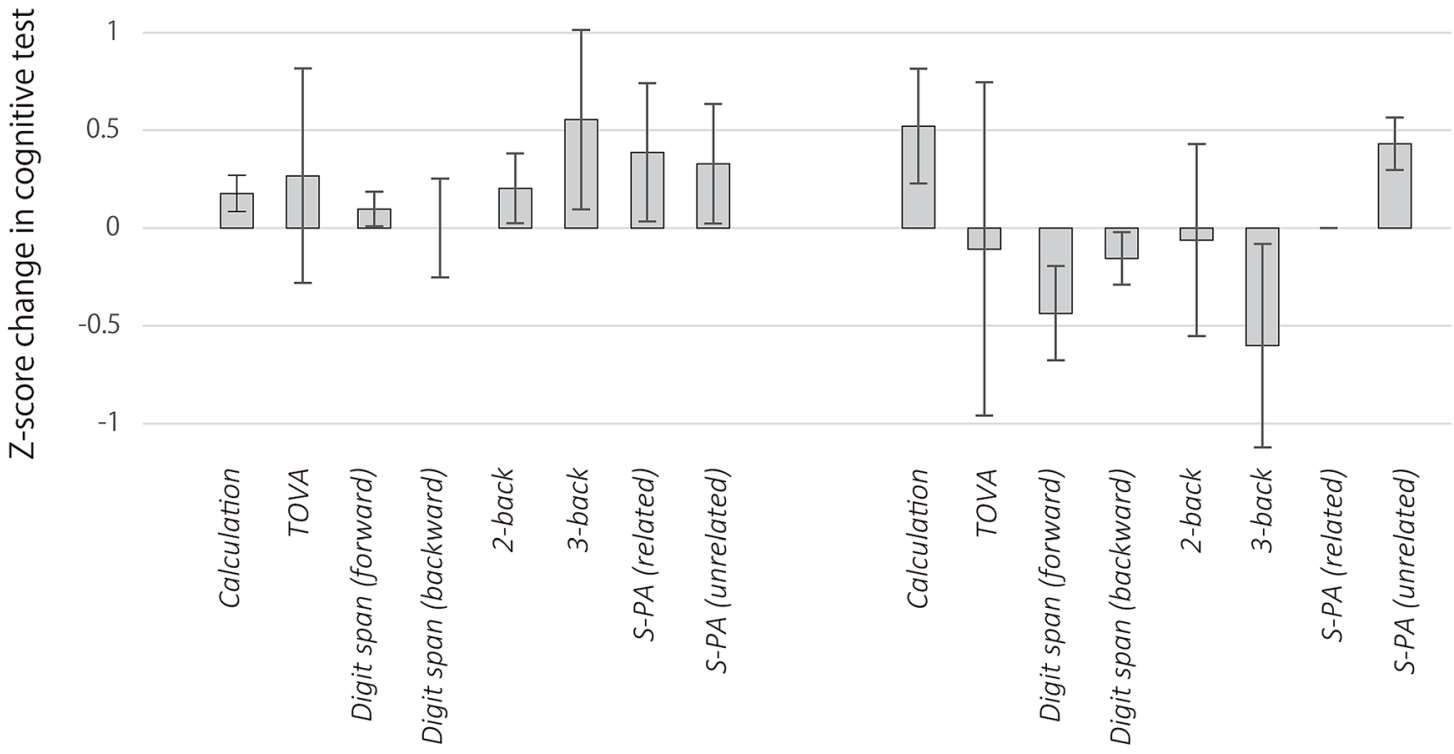

C

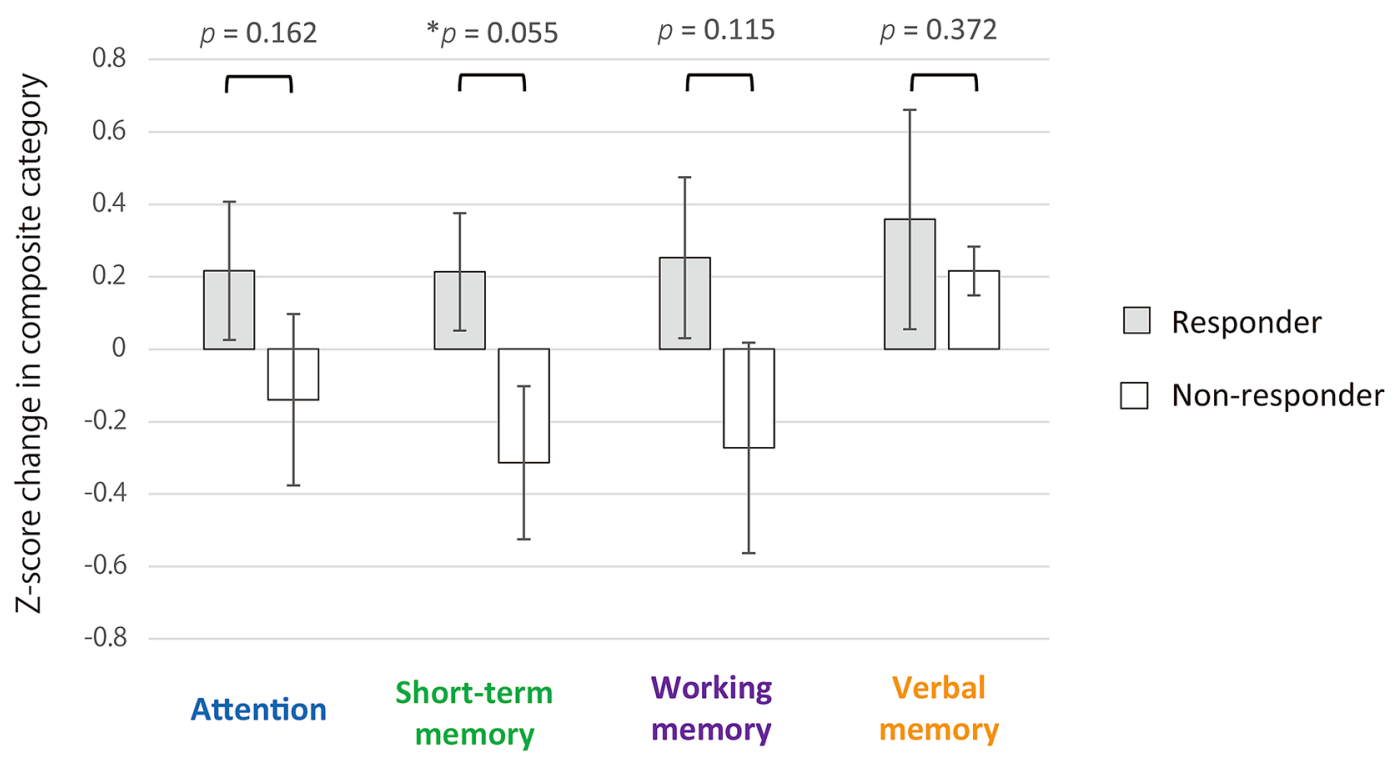


४Fig. 4 Comparison of changes in cognitive functions between responders and non-responders. Classification based on robust regression analysis revealed 6 responders and 4 non-responders (a). Changes in normalized cognitive test scores were compared between responders and non-responders (b). In each test, the change in the z-score was not significantly different between the two groups. A decrease was not observed in responders, whereas 5 out of 8 tests showed a decrease in non-responders. Changes in mean z-scores of each composite category were compared between responders and non-responders (c). In the four composite categories, only short-term memory showed significant difference

with and without a semantic relationship. The score of this task was expressed as the total number of correctly recalled words in three trials with the same set (Koike \& Sugishita, 2011). To avoid the learning effect, different batteries were used in the three evaluation periods.

In SCT, 250 questions consisting of four arithmetic operations using single-digit numbers were presented and the subject was asked to solve as many questions as possible in three minutes. The score of this task was the number of correct answers.

In NBT (Owen et al., 2005), 8 letters of the alphabet were presented by a PC using the open-source Matlab script, 'N-Back-for-Matlab' (Layden, 2018), in a random order with an interval of 1.8 or $2.0 \mathrm{~s}$ for two- and three-back tests, respectively. The subject was asked to push a key when the presented letter was the same as that presented two (twoback test) or three trials (three-back test) earlier. The twoback test was performed using 20 letters as a rehearsal. The two- and three-back tests were then performed using 40 letters each. The score of this task was provided by the above mentioned Matlab script, as described previously (Layden, 2018).

In TOVA, two types of figures, each of which has a black dot on the upper or lower sides of a white square, were displayed on a monitor at the same frequency for $20 \mathrm{~min}$. The subject was asked to push a button when a figure with a black dot on the upper side was presented. The TOVA score was estimated from the TOVA database based on the response speed, accuracy, and age (Forbes, 1998; Greenberg \& Waldman, 1993).

\section{Data Analysis of Cognitive Test Scores}

Since each subject performed the cognitive tests before and after the first neurofeedback period and after the second feedback period, we collected 30 scores from 10 subject regarding each cognitive test. To investigate the effects of neurofeedback on each test, the score of each subject was normalized using the mean and standard deviation of the 30 scores. Differences in scores between before and after feedback were considered to reflect the effects of neurofeedback on each test.
The tests of cognitive functions used in the present study were categorized into 4 composite categories (attention, short-term memory, working memory, and verbal memory), as shown in Fig. 2, by referring to the literature on methods to evaluate cognitive functions (Baddeley, 1992; Cullum, 1998; Kane et al., 2007; Owen et al., 2005; Reynolds, 1997) and instructions on sub-items of the established tests of cognitive functions, such as WAIS (D Wechsler, 1997; David Wechsler, 2008), WMS (David Wechsler, 1987), WISC (David Wechsler, 1991), the Standard Verbal Paired-Associate Learning Test (JSfHB, 2011), N-back test (Owen et al., 2005), and TOVA (Greenberg \& Waldman, 1993).

The mean $\mathrm{z}$-score among the cognitive tests was calculated in each composite category (Bird et al., 2019) and the scores of responders and non-responders were statistically compared using the Student's $t$-test $(\alpha=0.1)$. Prior to comparisons, the normality of the distribution was tested using the Shapiro-Wilk test (Royston, 1992).

\section{Results}

\section{Verification of Alpha Wave Neurofeedback}

nAP is normalized alpha-power calculated every three seconds based on the corresponding calibration data. By averaging all the time-series nAPs of three sessions in every feedback day, daily nAPs were obtained. According to the crossover design of the present study, the daily nAP of NF and RF across groups A and B were compared (Fig. 3a). Before the comparison, the normality of the distribution of each nAP was confirmed. As shown in Fig. 3b, the nAP of the late section was significantly higher in NF ( $p=0.0383)$, which suggested that constant neurofeedback increased the inducibility of the alpha wave in each session.

A crossover design generally has a washout period between the first and second interventions to avoid type 1 errors due to the carryover effect. However, the appropriate interval of the washout period is unknown in neurofeedback studies. In the present study, the first and second neurofeedback periods were separated by at least two days, which may not have been sufficient to eliminate the sustained effect of the first neurofeedback session. To elucidate the impact of the sustained effect, a longitudinal analysis of nAPs was performed in each group (Fig. 3c). Group A showed a slight increase in NF and a slight decrease in RF. In group A, nAP of the late section was significantly higher in NF than in RF ( $p=0.047)$. On the other hand, group B showed a slight decrease in both periods. No significant differences were observed in the nAPs of the late section between RF and NF; however, the latter was slightly higher. 


\section{Changes in Cognitive Function}

To evaluate cognitive test scores in association with responsiveness to neurofeedback, subjects were classified into responders and non-responders based on the results of a robust regression analysis of the time course of daily nAPs, yielding 6 responders (group A: 4 , group B: 2 ) and 4 nonresponders (group A: 1, group B: 3) (Fig. 4a).

Changes in normalized cognitive test scores were compared between responders and non-responders (Fig. 4b). In each test, the $\mathrm{z}$-score change was not significantly different between the two groups. A decrease was not observed in responders, whereas five out of eight tests showed a decrease in non-responders, suggesting that alpha wave neurofeedback improved a diverse spectrum of brain functions after 16 days of consecutive sessions.

Changes in the mean z-scores of each composite category were then compared between responders and non-responders (Fig. 4c). Prior to each comparison, the normality of the distribution of the scores of corresponding composite categories were confirmed in each group, except for verbal memory. In the four composite categories, short-term memory showed a significant improvement $(p=0.055)$.

\section{Discussion}

In the present study, ten subjects underwent NF and RF constantly for 32 days over more than two months. A crossover analysis revealed that normalized alpha-power was significantly higher in NF than in RF. This result demonstrated that constant auditory neurofeedback using music enabled subjects to easily induce the alpha wave, which was the primary end point of the present study. Auditory alpha neurofeedback was easy to continue and a promising protocol that warrants further development.

Changes in cognitive functions by neurofeedback were investigated as a secondary endpoint. A composite categorybased analysis revealed that the degree of improvement in short-term memory was significantly higher in responders than in non-responders. This result suggested that constant auditory alpha wave neurofeedback reinforced short-term memory.

Only a limited number of previous studies on music-based auditory alpha neurofeedback have been reported. Alexander et al. conducted a study using a single session of musical alpha neurofeedback for 16 subjects and reported improvement in their mental status (Alexander, 2018). Ramirez et al. showed that 5 weeks of alpha and beta neurofeedback training improved depressive condition in 6 elderly people (Ramirez et al., 2015). These studies successfully suggested neuro-modulatory effect of musical auditory alpha neurofeedback. The present study is of notable significance in that it validated the effectiveness of musical auditory alpha neurofeedback by adopting longer-term crossover design and showing an increase in alpha power as well as improvement in cognitive function.

\section{Time Course of Effects of Auditory Alpha Wave Neurofeedback}

The present results confirmed that auditory neurofeedback with a wearable EEG device for 16 days in 4-6 weeks enabled the augmentation of alpha-power. The majority of previous studies adopted a neurofeedback period ranging from a single session (Hanslmayr et al., 2005) to one week (Zoefel et al., 2011). With the aim of developing a neurofeedback system that may be constantly and casually performed, we validated a long-term neurofeedback system using the wearable EEG device. The entire feedback period consisted of NF and RF periods of 4-6 weeks each, lasting more than two months, which is one of the most constant protocols used in long-term neurofeedback studies. Daily normalized alpha-power in the NF period of group A was higher in the second two weeks than in the first, suggesting that a period of at least two weeks of neurofeedback is needed for effective alpha wave control using wearable EEG devices.

On the other hand, the longitudinal analysis of normalized alpha-power in each group suggested a sustained effect presumably due to preceding feedback. In group A, in which NF preceded RF, daily normalized alpha-power further increased in the early section of RF and decreased in the late section. This was assumed to be a sustained effect of a strategy developed for alpha wave control in preceding NF. In contrast, in group B in which RF preceded NF, the increase observed in daily normalized alpha-power was not significant. The unexpected decrease in the alpha power observed during NF sessions in group B might be caused by decline in subjects' motivation due to long-term feedback over 2 months. We used the same music throughout all sessions across the study, which could have negatively affected the result. Changing music in the course of feedback sessions or using relaxing one could be useful to maintain the neurofeedback effect longer (Phneah \& Nisar, 2017). Also, we could speculate that a wrong strategy developed during the preceding RF period might have canceled the effects of NF through the entire period. By taking the opposing effects of both NF and RF into consideration, the effects of onemonth-long neurofeedback, both positively and negatively, may have prolonged it for more than two weeks.

\section{Relationship Between Alpha Wave Control and Cognitive Functions}

Controversy surrounds the effects of alpha neurofeedback (Rogala et al., 2016) on cognitive functions due to 
negative findings (Naas et al., 2019). In the present study, the degree of improvement in short-term memory was higher in responders than in non-responders, which is consistent with previous findings showing an improvement in shortterm memory in association with alpha wave augmentation (Hsueh et al., 2016; Klimesch, 2012; Klimesch et al., 2006; Zoefel et al., 2011).

Although the theta wave has been a major topic of interest in terms of the relationship between memory and EEG, the alpha wave also plays a pivotal role in memory and a wide range of cognitive functions (Jensen et al., 2002; Klimesch, 2012). Klimesch et al. reported that the alpha wave was crucially involved in the inhibition and timing of neuronal activation, and provides accessibility to stored knowledge through fundamental functions of attention, such as suppression and selection. The improvement in short-term memory associated with an increased alpha wave in the present study appears to be consistent with these assumed roles of the alpha wave.

There are various advantages to using the alpha wave in long-term neurofeedback, which is supposed to be performed in daily life. The alpha wave is the most robust brain activity recorded by scalp EEG across a wide age range, from childhood to old age (Smit et al., 2012). A reliable alpha wave recording is easily verified by opening and closing the eyes. Due to the universality and manageability of the alpha wave, the finding of improved short-term memory appears to be promising, which encourages the further development of alpha wave neurofeedback systems.

It is important to note that the improvement observed in the score of the working memory category was slightly better in responders than in non-responders $(p=0.115)$. Working memory may improve with enhancements in short-term memory because visual and auditory short-term memory constitute the core of Baddeley's model of working memory (Baddeley, 1992). The lack of a significant improvement in working memory may have been due to the effect size and number of subjects in this study being selected to primarily validate the influence of the alpha wave neurofeedback system, which was the primary purpose of the present study.

\section{Advantages of the Wearable Neurofeedback System in the Present Study}

While an increasing number of studies on alpha wave neurofeedback using high-performance clinical EEG machines have been reported (Guez et al., 2015; Navarro Gil et al., 2018; Zoefel et al., 2011), few have employed a neurofeedback system using portable EEG devices (Stopczynski et al., 2014; Wei et al., 2017). Further studies are needed to establish an easy-to-use neurofeedback system utilizing portable, particularly wireless, and wearable EEG devices in order to promote the generalized use of neurofeedback in daily life.
The wearable device used in the present study has excellent device mobility and sufficient system specification. Furthermore, the software that automatically performs the analysis and feedback may be easily incorporated into a tablet device by each user. These characteristics pair perfectly with the musical auditory alpha wave feedback protocol used in the present study. It is encouraging that the combination achieved alpha wave augmentation and improved short-term memory. This may be helpful for children with ADHD and elderly individuals with mild cognitive impairment, who may require constant and long-term neurocognitive rehabilitation.

\section{Limitations}

It was difficult to recruit subjects who were able to fully cooperate such a constant and long-term protocol in the controlled condition, resulting in the small sample size of the present study. This preliminary result needs to be further validated in a larger study.

Also, we validated the feedback protocol by incorporating normal and random feedback sessions in a crossover design, without directly comparing each session to a control session in which subjects just listen to a music. This could be a confounding factor, which should be addressed in future study.

Since cognitive function tests were scheduled in the everyday life of subjects, it was difficult to exclude the effects of the physical and mental exhaustion of each day on the results of the tests. Therefore, the results of the cognitive tests included not only the effects of neurofeedback itself, but also considerable errors from the fluctuating state of mind of each subject. In other words, the present study reflects real-world circumstances, which is an important element in validating a system intended to be used in daily life.

\section{Conclusion}

In healthy subjects, an auditory alpha wave neurofeedback system with a novel music-based protocol using an easyto-use wearable EEG device was validated. A crossover analysis revealed that normalized alpha-power was significantly higher in NF than in RF, showing that constant neurofeedback with the system allowed subjects to easily induce the alpha wave. A composite category-based analysis of cognitive functions revealed that the degree of improvement in short-term memory was higher in responders than in non-responders. The present study adopted one of the most constant protocols used in previous long-term neurofeedback studies and achieved successful neurofeedback in terms of alpha wave control and cognitive function. This is encouraging for children with ADHD and elderly individuals with 
mild cognitive impairment, who may require constant and long-term neurocognitive rehabilitation.

Acknowledgements This research was conducted with support from Mediaseek Inc., which provided the tablet devices with the analysis application and the wearable EEG devices used in the present study during the research period.

Author Contributions NK, SS, and HN conceptualized this research. KT performed data curation. KT analyzed data. KT, NK, SS, MT, KY, and $\mathrm{HN}$ interpreted data. KT and NK drafted the manuscript. HN and NS supervised and revised the manuscript.

Funding This research was supported in part by a Grant-in-Aid (No. 19K09452) for Scientific Research (C) from the Japan Society for the Promotion of Science, and a research grant from Kanae Foundation for the Promotion of Medical Science.

Data Availability Due to the nature of this research, participants of this study did not agree for their data to be shared publicly, so supporting data is not available.

\section{Declarations}

Conflict of interest The authors declare that there is no financial conflict of interest. Mediaseek Inc. provided the tablet devices with the analysis application and the wearable EEG devices used in the present study during the research period.

Ethical Approval The present study was approved by the Research Ethics Committee, Graduate School of Medicine and Faculty of Medicine, the University of Tokyo (\#2018077NI).

Consent for Participate Informed consent was obtained from all individual participants included in the study.

Consent for Publication The participant has consented to the submission of the research to the journal.

Open Access This article is licensed under a Creative Commons Attribution 4.0 International License, which permits use, sharing, adaptation, distribution and reproduction in any medium or format, as long as you give appropriate credit to the original author(s) and the source, provide a link to the Creative Commons licence, and indicate if changes were made. The images or other third party material in this article are included in the article's Creative Commons licence, unless indicated otherwise in a credit line to the material. If material is not included in the article's Creative Commons licence and your intended use is not permitted by statutory regulation or exceeds the permitted use, you will need to obtain permission directly from the copyright holder. To view a copy of this licence, visit http://creativecommons.org/licenses/by/4.0/.

\section{References}

Alexander, F. (2018). Stress coping via musical neurofeedback. Advances in Mind Body Medicine, 32(2), 17-20.

Baddeley, A. (1992). Working memory. Science, 255(5044), 556-559.

Bergstrom, I., Seinfeld, S., Arroyo-Palacios, J., Slater, M., \& SanchezVives, M. V. (2014). Using music as a signal for biofeedback.
International Journal of Psychophysiology, 93(1), 140-149. https://doi.org/10.1016/j.ijpsycho.2013.04.013.

Bird, L. J., Jackson, G. D., \& Wilson, S. J. (2019). Music training is neuroprotective for verbal cognition in focal epilepsy. Brain, 142(7), 1973-1987. https://doi.org/10.1093/brain/awz124.

Biswas, A., \& Ray, S. (2019). Alpha Neurofeedback Has a Positive Effect for Participants Who Are Unable to Sustain Their Alpha Activity. eneuro, 6(4), ENEURO.0498-0418.2019. doi:https://doi. org/10.1523/ENEURO.0498-18.2019

Brancatisano, O., Baird, A., \& Thompson, W. F. (2020). Why is music therapeutic for neurological disorders? The therapeutic music capacities model. Neuroscience \& Biobehavioral Reviews, 112, 600-615. https://doi.org/10.1016/j.neubiorev.2020.02.008.

Bucho, T., Caetano, G., Vourvopoulos, A., Accoto, F., Esteves, I., Badia, S. B. i. Figueiredo, P. (2019, 23-27 July 2019). Comparison of Visual and Auditory Modalities for Upper-Alpha EEGNeurofeedback *. Paper presented at the 2019 41st Annual International Conference of the IEEE Engineering in Medicine and Biology Society (EMBC).

Cho, M. K., Jang, H. S., Jeong, S.-H., Jang, I.-S., Choi, B.-J., \& Lee, M.-G. T. (2008). Alpha neurofeedback improves the maintaining ability of alpha activity. NeuroReport, 19(3).

Choi, S. W., Chi, S. E., Chung, S. Y., Kim, J. W., Ahn, C. Y., \& Kim, H. T. (2011). Is alpha wave neurofeedback effective with randomized clinical trials in depression? A pilot study. Neuropsychobiology, 63(1), 43-51. https://doi.org/10.1159/000322290.

Cullum, C. M. (1998). Neuropsychological Assessment of Adults. In A. S. Bellack \& M. Hersen (Eds.), Comprehensive Clinical Psychology (pp. 303-347). Oxford: Pergamon

Duric, N., Assmus, J., Gundersen, D., \& Elgen, I. (2012). Neurofeedback for the treatment of children and adolescents with ADHD: a randomized and controlled clinical trial using parental reports. BMC Psychiatry, 12, 107. https://doi.org/10.1186/ 1471-244X-12-107.

Escolano, C., Navarro-Gil, M., Garcia-Campayo, J., \& Minguez, J. (2014). The effects of a single session of upper alpha neurofeedback for cognitive enhancement: A sham-controlled study. Applied Psychophysiology and Biofeedback, 39(3), 227-236. https://doi.org/10.1007/s10484-014-9262-9.

Fedotchev, A., Radchenko, G., \& Zemlianaia, A. (2018). On one approach to health protection: Music of the brain. Journal Integrative of Neuroscince, 17(3-4), 309-315. https://doi.org/10.3233/ JIN-170053.

Fernández, T., Bosch-Bayard, J., Harmony, T., Caballero, M. I., DíazComas, L., Galán, L., \& Otero-Ojeda, G. (2016). Neurofeedback in learning disabled children: Visual versus auditory reinforcement. Applied Psychophysiology and Biofeedback, 41(1), 27-37. https://doi.org/10.1007/s10484-015-9309-6.

Forbes, G. B. (1998). Clinical utility of the Test of Variables of Attention (TOVA) in the diagnosis of attention-deficit/hyperactivity disorder. Journal of Clinical Psychology, 54(4), 461-476. https://doi. org/10.1002/(sici)1097-4679(199806)54:4\%3c461::aid-jclp8\% 3e3.0.co;2-q.

Gevensleben, H., Holl, B., Albrecht, B., Schlamp, D., Kratz, O., Studer, P., \& Heinrich, H. (2010). Neurofeedback training in children with ADHD: 6-month follow-up of a randomised controlled trial. European Child \& Adolescent Psychiatry, 19(9), 715-724. https://doi. org/10.1007/s00787-010-0109-5.

Giofre, D., Stoppa, E., Ferioli, P., Pezzuti, L., \& Cornoldi, C. (2016). Forward and backward digit span difficulties in children with specific learning disorder. Journal of Clinical and Experimental Neuropsychology, 38(4), 478-486. https://doi.org/10.1080/13803 395.2015.1125454.

Greenberg, L. M., \& Waldman, I. D. (1993). Developmental normative data on the test of variables of attention (TOVA). Journal of Child 
Psychology and Psychiatry, 34(6), 1019-1030. https://doi.org/10. 1111/j.1469-7610.1993.tb01105.x.

Gruzelier, J. H. (2014). EEG-neurofeedback for optimising performance. I: a review of cognitive and affective outcome in healthy participants. Neuroscience \& Biobehavioural Reviews, 44, 124 141. https://doi.org/10.1016/j.neubiorev.2013.09.015.

Guez, J., Rogel, A., Getter, N., Keha, E., Cohen, T., Amor, T., \& Todder, D. (2015). Influence of electroencephalography neurofeedback training on episodic memory: A randomized, sham-controlled, double-blind study. Memory, 23(5), 683-694. https://doi. org/10.1080/09658211.2014.921713.

Hanslmayr, S., Sauseng, P., Doppelmayr, M., Schabus, M., \& Klimesch, W. (2005). Increasing individual upper alpha power by neurofeedback improves cognitive performance in human subjects. Applied Psychophysiology Biofeedback, 30(1), 1-10. https://doi. org/10.1007/s10484-005-2169-8.

Hsueh, J.-J., Chen, T.-S., Chen, J.-J., \& Shaw, F.-Z. (2016). Neurofeedback training of EEG alpha rhythm enhances episodic and working memory. Human Brain Mapping, 37(7), 2662-2675. https:// doi.org/10.1002/hbm.23201.

Jebelli, H., Hwang, S., \& Lee, S. (2018). EEG Signal-processing framework to obtain high-quality brain waves from an off-theshelf wearable EEG device. Journal of Computing in Civil Engineering, 32(1), 04017070. https://doi.org/10.1061/(ASCE)CP. 1943-5487.0000719.

Jensen, O., Gelfand, J., Kounios, J., \& Lisman, J. E. (2002). Oscillations in the Alpha Band $(9-12 \mathrm{~Hz})$ increase with memory load during retention in a short-term memory task. Cerebral Cortex, 12(8), 877-882. https://doi.org/10.1093/cercor/12.8.877.

Jirayucharoensak, S., Israsena, P., Pan-Ngum, S., Hemrungrojn, S., $\&$ Maes, M. (2019). A game-based neurofeedback training system to enhance cognitive performance in healthy elderly subjects and in patients with amnestic mild cognitive impairment. Clinical Interventions in Aging, 14, 347-360. https://doi.org/10.2147/ CIA.S189047.

JSfHB. (2011). Standard Verbal Paired-Associate Learning Test.

Kane, M. J., Conway, A. R. A., Miura, T. K., \& Colflesh, G. J. H. (2007). Working memory, attention control, and the N-back task: a question of construct validity. Journal of experimental psychology. Learning, memory, and cognition, 33(3), 615-622. https:// doi.org/10.1037/0278-7393.33.3.615.

Klimesch, W. (2012). Alpha-band oscillations, attention, and controlled access to stored information. Trends in Cognitive Sciences, 16(12), 606-617. https://doi.org/10.1016/j.tics.2012.10.007.

Klimesch, W., Doppelmayr, M., \& Hanslmayr, S. (2006). Upper alpha ERD and absolute power: Their meaning for memory performance. Event-Related Dynamics of Brain Oscillations, 159, 151-165. https://doi.org/10.1016/s0079-6123(06)59010-7.

Koike, A., \& Sugishita, M. (2011). The Japanese version of the Wechsler Memory Scale-revised. Nihon Rinsho, 69(Suppl 8), 408-412.

Lau-Zhu, A., Lau, M. P. H., \& McLoughlin, G. (2019). Mobile EEG in research on neurodevelopmental disorders: Opportunities and challenges. Developmental Cognitive Neuroscience, 36, 100635. https://doi.org/10.1016/j.dcn.2019.100635.

Lavy, Y., Dwolatzky, T., Kaplan, Z., Guez, J., \& Todder, D. (2019). Neurofeedback improves memory and peak alpha frequency in individuals with mild cognitive impairment. Applied Psychophysiology Biofeedback, 44(1), 41-49. https://doi.org/10.1007/ s10484-018-9418-0.

Layden, E. A. (2018). N-Back for Matlab (Publication no. https://doi. org/https://doi.org/10.12751/g-node.f87128).

Lofthouse, N., Arnold, L. E., Hersch, S., Hurt, E., \& DeBeus, R. (2012). A review of neurofeedback treatment for pediatric ADHD. Journal of Attention Disorders, 16(5), 351-372. https://doi.org/ $10.1177 / 1087054711427530$.
Maes, P. J., Buhmann, J., \& Leman, M. (2016). 3Mo: A model for music-based biofeedback. Frontiers in Neuroscience, 10, 548. https://doi.org/10.3389/fnins.2016.00548.

Markiewcz, R. (2017). The use of EEG Biofeedback/Neurofeedback in psychiatric rehabilitation. Psychiatria Polska, 51(6), 1095-1106. https://doi.org/10.12740/pp/68919.

Mihara, M., Miyai, I., Hattori, N., Hatakenaka, M., Yagura, H., Kawano, T., \& Kubota, K. (2012). Neurofeedback using real-time near-infrared spectroscopy enhances motor imagery related cortical activation. PLOS ONE, 7(3), e32234. https://doi.org/10.1371/ journal.pone.0032234.

Naas, A., Rodrigues, J., Knirsch, J. P., \& Sonderegger, A. (2019). Neurofeedback training with a low-priced EEG device leads to faster alpha enhancement but shows no effect on cognitive performance: A single-blind, sham-feedback study. PLoS ONE, 14(9), e0211668. https://doi.org/10.1371/journal.pone.0211668.

Namazi, H., \& Kulish, V. (2012). Mathematical modeling of human brain neuronal activity in the absence of external stimuli. Journal of Medical Imaging and Health Informatics, 2, 400-407. https://doi.org/10.1166/jmihi.2012.1112.

Navarro Gil, M., Escolano Marco, C., Montero-Marín, J., Minguez Zafra, J., Shonin, E., \& García Campayo, J. (2018). Efficacy of neurofeedback on the increase of mindfulness-related capacities in healthy individuals: A controlled trial. Mindfulness, 9(1), 303-311. https://doi.org/10.1007/s12671-017-0775-1.

Nawaz, R., Nisar, H., \& Voon, Y. V. (2018). The Effect of Music on Human Brain; Frequency Domain and Time Series Analysis Using Electroencephalogram. Ieee Access, 6, 45191-45205. https://doi.org/10.1109/Access.2018.2855194.

Owen, A. M., McMillan, K. M., Laird, A. R., \& Bullmore, E. (2005). N-back working memory paradigm: A meta-analysis of normative functional neuroimaging studies. Human Brain Mapping, 25(1), 46-59. https://doi.org/10.1002/hbm.20131.

Phneah, S. W., \& Nisar, H. (2017). EEG-based alpha neurofeedback training for mood enhancement. Australas Physical and Engineering Sciences in Medicine, 40(2), 325-336. https://doi.org/ 10.1007/s13246-017-0538-2.

Ramirez, R., Palencia-Lefler, M., Giraldo, S., \& Vamvakousis, Z. (2015). Musical neurofeedback for treating depression in elderly people. Frontiers in Neuroscience. https://doi.org/10.3389/ fnins.2015.00354.

Reynolds, C. R. (1997). Forward and backward memory span should not be combined for clinical analysis. Archives of Clinical Neuropsychology, 12(1), 29-40. https://doi.org/10.1016/S08876177(96)00015-7.

Rogala, J., Jurewicz, K., Paluch, K., Kublik, E., Cetnarski, R., \& Wróbel, A. (2016). The do's and don'ts of neurofeedback training: a review of the controlled studies using healthy adults. Frontiers in Human Neuroscience, 10, 301-301. https://doi.org/ 10.3389/fnhum.2016.00301.

Royston, P. (1992). Approximating the Shapiro-Wilk W-test for nonnormality. Statistics and Computing, 2(3), 117-119. https://doi. org/10.1007/BF01891203.

Schoenberg, P. L., \& David, A. S. (2014). Biofeedback for psychiatric disorders: a systematic review. Applied Psychophysiology and Biofeedback, 39(2), 109-135. https://doi.org/10.1007/ s10484-014-9246-9.

Schönenberg, M., Wiedemann, E., Schneidt, A., Scheeff, J., Logemann, A., Keune, P. M., \& Hautzinger, M. (2017). Neurofeedback, sham neurofeedback, and cognitive-behavioural group therapy in adults with attention-deficit hyperactivity disorder: a triple-blind, randomised, controlled trial. The Lancet Psychiatry, 4(9), 673-684. https://doi.org/10.1016/S2215-0366(17) 30291-2.

Smit, D. J. A., Boersma, M., Schnack, H. G., Micheloyannis, S., Boomsma, D. I., Hulshoff Pol, H. E., \& de Geus, E. J. C. (2012). 
The brain matures with stronger functional connectivity and decreased randomness of its network. PLoS ONE, 7(5), e36896. https://doi.org/10.1371/journal.pone.0036896.

Stopczynski, A., Stahlhut, C., Petersen, M. K., Larsen, J. E., Jensen, C. F., Ivanova, M. G., \& Hansen, L. K. (2014). Smartphones as pocketable labs: visions for mobile brain imaging and neurofeedback. International Journal Psychophysiology, 91(1), 54-66. https:// doi.org/10.1016/j.ijpsycho.2013.08.007.

Sulzer, J., Haller, S., Scharnowski, F., Weiskopf, N., Birbaumer, N., Blefari, M. L., \& Sitaram, R. (2013). Real-time fMRI neurofeedback: progress and challenges. NeuroImage, 76, 386-399. https:// doi.org/10.1016/j.neuroimage.2013.03.033.

van Albada, S. J., \& Robinson, P. A. (2013). Relationships between electroencephalographic spectral peaks across frequency bands. Frontiers in Human Neuroscience, 7, 56. https://doi.org/10.3389/ fnhum.2013.00056.

van Boxtel, G. J., Denissen, A. J., Jäger, M., Vernon, D., Dekker, M. K., Mihajlović, V., \& Sitskoorn, M. M. (2012). A novel self-guided approach to alpha activity training. International Journal Psychophysiology, 83(3), 282-294. https://doi.org/10.1016/j.ijpsy cho.2011.11.004.

Wechsler, D. (1987). WMS-R: Wechsler memory scale-revised: Psychological Corporation.

Wechsler, D. (1991). WISC-III: Wechsler intelligence scale for children: Manual: Psychological Corporation.

Wechsler, D. (1997). Wechsler Adult Intelligence Scale-III. 1997. San Antonio: The Psychological Corporation.

Wechsler, D. (2008). Wechsler adult intelligence scale-Fourth Edition (WAIS-IV). San Antonio, TX: NCS Pearson, 22(498), 816-827.
Wei, T. Y., Chang, D. W., Liu, Y. D., Liu, C. W., Young, C. P., Liang, S. F., \& Shaw, F. Z. (2017). Portable wireless neurofeedback system of EEG alpha rhythm enhances memory. BioMedical Engineering OnLine, 16(1), 128. https://doi.org/10.1186/s12938-017-0418-8.

Woods, D. L., Kishiyamaa, M. M., Lund, E. W., Herron, T. J., Edwards, B., Poliva, O., \& Reed, B. (2011). Improving digit span assessment of short-term verbal memory. Journal of Clinical and Experimental Neuropsychology, 33(1), 101-111. https://doi.org/ 10.1080/13803395.2010.493149.

Yamin, H. G., Gazit, T., Tchemodanov, N., Raz, G., Jackont, G., Charles, F., \& Cavazza, M. (2017). Depth electrode neurofeedback with a virtual reality interface. Brain-Computer Interfaces, 4(4), 201-213. https://doi.org/10.1080/2326263X.2017.1338008.

Yeh, W. H., Hsueh, J. J., \& Shaw, F. Z. (2020). Neurofeedback of alpha activity on memory in healthy participants: a systematic review and meta-analysis. Frontiers in Human Neuroscience, 14, 562360. https://doi.org/10.3389/fnhum.2020.562360.

Zoefel, B., Huster, R. J., \& Herrmann, C. S. (2011). Neurofeedback training of the upper alpha frequency band in EEG improves cognitive performance. NeuroImage, 54(2), 1427-1431. https://doi. org/10.1016/j.neuroimage.2010.08.078.

Publisher's Note Springer Nature remains neutral with regard to jurisdictional claims in published maps and institutional affiliations. 